\title{
Energy Efficient Two-hop D2D Communications Underlay 5G Networks: A Stackelberg Game Approach
}

\author{
Sawsan Selmi, Member, IEEE, and Ridha Boullègue, Member, IEEE
}

\begin{abstract}
Although coverage and capacity are the key elements of the $5 \mathrm{G}$ user experience, a dominant part of the population living in rural areas still experience inferior connectivity. Several solutions have been proposed to address this issue. They include deploying small cells, increasing the number of sectors per eNodeB, and reusing signal repetition. However, most of them require complex deployment and expensive fees. Accordingly, many efforts have been deployed on coverage extension software. Even so, many critical issues related to public safety, relay capacity, and devices power constraints are still challenging. As a contribution, we propose in this paper a spectral and energy-efficient two-hop device to device (D2D) relay selection algorithm. Our main goal is to extend the connectivity to the out-of-coverage (OOC) devices. Contrarily to previous solutions in which the relay is selected centrally or individually, we propose a distributed two-stage algorithm based on the Stackelberg game to involve all the competing devices. In the first stage, the OOC devices (OCDUs) are matched with the relays maximizing their spectral efficiency, and the required bandwidth for each one is determined. Then, a power control stage is investigated to calculate the optimal transmission power. The numerical and simulation analysis shows that the proposed schema outperforms the former solutions in total system capacity, spectral efficiency (SE), and energy efficiency (EE) while reducing the complexity.
\end{abstract}

Index Terms-Coverage extension, two-hop D2D, energy efficiency, spectral efficiency, Stackelberg game.

\section{INTRODUCTION}

$\mathrm{R}$ ecently, with the overload of the conventional cellular system, the new generation mobile networks face several issues related to the increasing demands for ubiquitous connectivity and the exponential volume of data traffic. Accordingly, device-to-device communication was integrated

Manuscript received January 19, 2021; revised March 5, 2021. Date of publication March 25, 2021. Date of current version March 25, 2021. The associate editor prof. Pascal Lorenz has been coordinating the review of this manuscript and approved it for publication.

Sawsan Selmi is with the Innov'Com Laboratory, National School of Engineers of Tunis, University of Tunis El Manar, Tunisia (e-mail: sawssan.selmi@gmail.com). Ridha Bouallègue is with the Innov'Com Laboratory, Higher School of Communications of Tunis, Carthage University, Tunisia (e-mail: ridha.bouallegue@gmail.com).

A part of this paper was presented at the International Conference on Software, Telecommunications and Computer Networks (SoftCOM) 2020.

Digital Object Identifier (DOI): 10.24138/jcomss-2021-0008 as a key technology in the $5 \mathrm{G}$ networks to enable direct communication between close devices instead of conveying their data through the core network [1]. Its benefits include offloading the traffic from the eNodeB, enhancing the system capacity, the energy efficiency [2], and decreasing the latency due to the short signal traversal path [3]. However, when it comes to coverage extension, a number of issues arise. According to ITU (International Union Telecommunications) 2018 reports, more than 4 billion people, representing $45 \%$ of the world's population, especially those living in rural and maritime areas are not connected; 5G network is targeted primarily at densely populated areas and depends on tightly deployed small cells [4]. So it is unlikely to replace the $4 \mathrm{G}$ for coverage outside towns and cannot be a good solution for the digital divide that affects those areas.

Face to this situation, several solutions were proposed such as deploying small cells, increasing the number of sectors per eNodeB, and reusing signal repetition mechanisms. However, most of them did not properly improve the uplink relaying. More importantly, they did not provide the connectivity in public safety scenarios or any other situations with partial coverage. Meanwhile, they require additional network deployment; energy and maintenance costs and did not consider the device's power and relay capacity.

To manage these issues, Release 15 3GPP added many enhancements to Release 13 UE-to-network relaying to support end-to-end security and QoS as well as efficient path between conventional and D2D interfaces. In addition, the needed changes for sidelink were studied to provide a reliable D2D communication link for low cost and low power devices. In this scenario; a remote device can access the network through the assistance of a relay using outbound or in band communication. In the former, the devices use unlicensed spectrum as Bluetooth and $\mathrm{WiFi}$ to communicate with the relay [5], whereas the latter utilizes the cellular RBs in overlay or underlay cellular mode. The underlay mode enhances the cellular capacity and the overall spectral efficiency. However, it requires more work on the dynamic resource allocation and relay selection [6].

Several schemas have been proposed to manage the relay selection in underlay mode. Most of them has been done centrally or individually per device; and did not consider the current state of the network such as the devices density, 
locations, their transmission power and the current channel conditions. In this paper, we derive means for getting optimal QoS for all the devices in the system leveraging from the Stackelberg game. The utility function captures the network information such as the links gain, the signal-to-interferenceplus-noise-ratios, the spectral and energy efficiency. At the equilibrium, the optimal relay and transmission power are determined for each OCDU considering all the other devices preferences.

In our previous work we investigated a joint SE/EE multihop communication based on D2D clusters [7]. The relay selection algorithm enhances the spectrum reuse and controls the energy consumption inside the cell. In this paper, we extend the former schema to the out of coverage devices. Our main goal is to select the best relay requiring the minimum energy consumption and providing the maximum spectral efficiency to the out of coverage UEs. Besides, this approach preserves the QoS of the primary cellular communications.

To organize our work, we structured the rest of the paper as follows. In Section II, we analyze the main issues related to the existing work on coverage extension. In section III, we present the main contributions of the proposed approach. The system model of the two-hop D2D communication applied to the proposed algorithm is presented in section IV. Section V models the resource allocation and the relay selection problems through a Stackelberg game. Section VI describes the distributed spectral and energy-efficient algorithm. In Section VII, the performances of the proposed approach are evaluated and compared to the former schemas. Finally, Section VIII concludes the paper and presents some perspectives for future work.

\section{RELATED WORKS}

Since Release 13 3GPP, an out of coverage UE can communicate with the eNodeB through another device, acting as a temporary wireless relay. Various optimization approaches have been applied to provide the maximum coverage at the cell edge, based on power control, interference minimization, and relay selection.

In this section, we study the main solutions of two-hop D2D communication in partial coverage scenarios, where devices located in non-covered areas can benefit from other UEs acting as extensions of active cells [9]. Meanwhile, the candidate relay may experience interference, require more energy resources, and calculation capabilities to route the additional traffic.

Many papers propose clustering approaches $[8,9,10]$. They group the covered mobile terminals in clusters then a cluster head is selected to relay the data to the out of coverage UE. This architecture reduces the device's energy consumption, guarantees the quality and the flow of the communication process. However, there are still some related problems. It requires frequent switching in the cluster head because it cannot support multiple devices simultaneously and continuously due to its limited battery capacity. To tackle this problem, reference [11] proposed a power control mechanism to select the relay with the maximum remaining energy. However, it did not manage the interference generated by the relays' power to the co-channel devices.
Papers [12,13] use the traversal method to cluster terminals. However, the computational complexity is high. Furthermore, they did not consider the impact of the power consumption in the relay selection process.

Authors of paper [14] investigate a power control schema, where the relay requiring lowest energy transmission from other devices is privileged. This algorithm can achieve good performance when relaying one device. Yet, it cannot be adapted if many users share the same relay.

Other distributed approaches based on game theory studied how the relays interact with the D2D devices and route their data to the eNodeB in an asymmetric mode. Authors of Reference [15] present a game-theoretic scheme whereas, in reference [16], they proposed a pricing game to stimulate cooperative diversity among non-cooperative nodes.

The authors of reference [28] model the relay selection problem through a cooperative game to maximize the devices rate. However, the energy consumption was not studied too. Auction theory is another state-of-the-art approach based on pricing models $[17,18]$. In References $[19,20]$, a buyer/seller game was proposed to perform the power allocation in cooperative transmission. The authors formulated the game as a buyers' market competition where multiple relays compete to gain the highest profit from selling resource blocks to the out of coverage devices. The first situation consists of one data buyer purchasing from several data sellers. The second model consists of one seller selling data to multiple buyers. In [21], the authors use the combinatorial auction theory to relay outof-coverage devices in a single-relay scenario. However, they did not study the power consumption [22-23].

Paper [24] considers a cooperative transmission for wireless Ad-Hoc networks. The authors formulate the problem as a Stackelberg game to share the relay resources with a set of covered sources. They used the binary non-coherent frequency shift keying (2FSK) in OFDM. However, multiplayers and relays scenario is not considered.

Paper [25] investigated a joint spectral and power management algorithm for multi-user mobile edge. The proposed schema uses a stochastic approach to minimize the energy consumption. However, the computational complexity is high, and the number of devices to be relayed must be known in advance. Paper [26] proposes a two-stage approach. First, a weighted sum maximum algorithm is investigated to select the best relay. Then, a random communication time is allocated.

The energy-efficient schema proposed in [27] manages the power allocation of D2D transmitter and relays. Yet, the multi-relays scenario was not considered too. In [28], an energy-efficient relay selection is studied based on the power allocation. However, a single relay scenario is investigated. Paper [29] proposes a power allocation and relay selection schema for relay-assisted D2D.Nevertheless, fading channels, multi-user scenario and transmitters are not analyzed.

\section{THE MAIN CONTRIBUTIONS}

In this paper, we propose a novel distributed algorithm to extend the data transmission and improve the system spectral and energy efficiency. The system aims to maximize the number of supported out-of-coverage D2D users while preserving the Quality of Service (QoS) of the relays. 
We formulate the relay selection problem as a Stackelberg game and decompose it into two stages to approach the game equilibrium. The first stage selects the optimal relay to the outof-coverage DUE, and the second controls the transmission power. The D2D users communicate in an underlay mode; they share the radio resources with the cellular uplink communications.

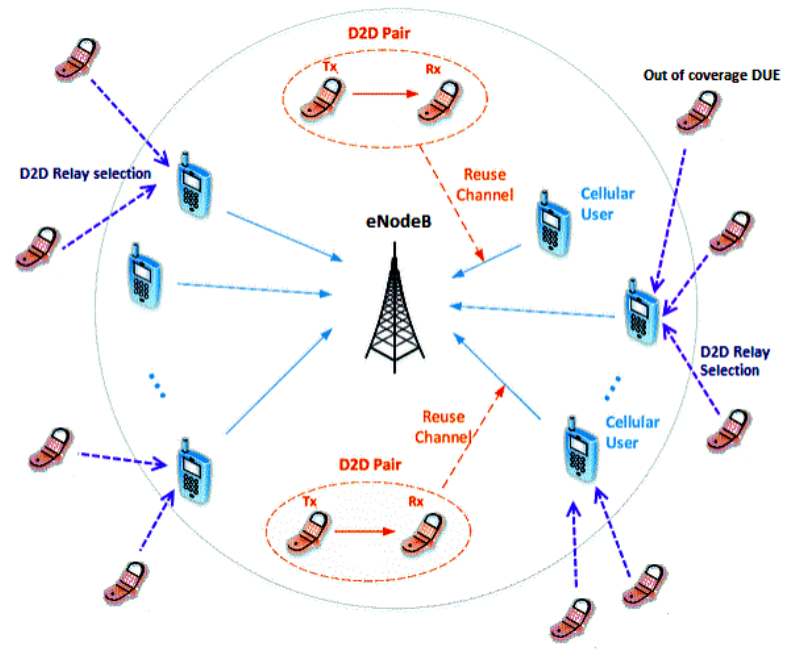

Fig. 1. System Model of the two-hop D2D communication

The network throughput and spectral efficiency is further improved by exploiting multiuser diversity; anOCDU can reuse resources from different relays and a relay RB can be allocated to multiple DUEs. The Stackelberg game is used to model the interaction between the relays and OCDUs. Given the bandwidth cost, the relay can share the resource based on the communication channel condition and co-channel interference.

We use the fractional programming to transform the non convex problem into a convex optimization problem. Then, we propose an optimization iterative algorithm to solve the convex optimization problem. Finally, the equilibrium of the game is reached at the maximum utility. Simulation results show that the proposed schema enhances the total capacity, spectral and energy efficiency with a relatively simple calculation and tractable results, compared to the existing solutions.

\section{SYSTEM MODEL}

We consider a partial coverage scenario where an out of coverage device can communicate with the eNodeB through another device acting as a temporary wireless relay. The system has $\mathrm{N}$ relays and M OCDUs. A relay communicates directly with the eNodeB through an uplink cellular channel. Thus, there are in total $\mathrm{N}$ uplink channels. Each relay shares its resource blocks (RBs) with many OCDUs, to maximize the cellular capacity. Meanwhile, the relays cellular transmission is more prioritized than relayed data. To minimize the cochannel interference, the maximum D2D distance and transmission power are constrained by $P_{d m a x}$ and $R_{d}$ respectively.

Figure 1 presents the system model of the two-hop D2D coverage extension scenario. A node $R_{i}$ closer to the cell edge and with a good channel condition is designated as a potential relay to assist the out of coverage transmitters. Let $i=$ $\left\{R_{1}, R_{2}, \ldots, R_{N}\right\}$ be the set of relays in the system. $D U E_{1}, D U E_{2}$, $\ldots, D U E_{M}$ are competing to share these resources in an underlay mode. Co-channel interference can occur between the relay communication and the devices sharing the same resource bloc. Yet, there is no inter-cell interference among relays due to the OFDMA orthogonality.

\section{PROBLEM FORMULATION}

In the following, we first outline the process of cooperative transmission. In the literature, relaying strategies include two main categories: Decode-and-Forward (DF) and Amplify-andForward (AF). DF maximizes the spectrum efficiency. Yet, it raises the probability of errors, especially when the devices are in motion. AF is simple to implement. It outperforms $\mathrm{DF}$ in coverage extension and capacity particularly in dense networks with significant shadowing [18]. Besides it does not require processing hardware as other relying strategies. $\mathrm{AF}$ can have a fixed or variable gain. The first applies a constantcoefficient to the received signal from the source-relay amplitude hop. While the second ensures an accurate output power by inverting the channel input.

In the proposed schema, the AF strategy is the most applicable. We consider a half duplex mode with two-phases. In the first, the source $S$ transmits $x$ symbols to the relay $R$. In the second, the relay broadcasts a scaled combination of the received signal. Finally, the destination subtracts or cancels the self-interference and extracts the target signal.

The received signals $Y_{S D}$ and $Y_{R}$ at the destination and the relay are given respectively by:

$$
\begin{gathered}
Y_{R}=h_{S R} x+n_{R}(1) \\
Y_{S D}=h_{S D} x+n_{D}(2)
\end{gathered}
$$

where $n_{R} \sim C N\left(0, \sigma_{R}^{2}\right), n_{D} \sim C N\left(0, \sigma_{D}^{2}\right)$ are the additive white Gaussian noise (AWGN) at the relay and destination respectively. $h_{S R}$ and $h_{S D}$ are the channel fading coefficients of the source-relay, and source-destination respectively. To satisfy the constraint of relay power $P_{R}$, the relay gains its received message by the scaling factor:

$$
\beta=\sqrt{\frac{P_{r}}{P_{S}\left|h_{S R}\right|^{2}+\sigma_{R}^{2}}}(3)
$$

where $P_{S}$ is the source power. Hence the symbol received at the destination in the second phase is determined by:

$$
Y_{R D}=h_{R D} \beta Y_{R}+n_{D}=\sqrt{\frac{P_{r}}{P_{S}\left|h_{S R}\right|^{2}+\sigma_{D}^{2}}} h_{S R} h_{R D} x+n_{D}^{\prime}(4)
$$

where $h_{R D}$ represents the channel coefficient between the relay and the destination, and

$$
n_{D}=\sqrt{\frac{P_{r}}{P_{S}\left|h_{S R}\right|^{2}+\sigma_{D}^{2}}} h_{R D} n_{R}
$$

is also an AWGN with a variance: 


$$
\sigma=\frac{P_{r}\left|h_{R D}\right|^{2} \sigma_{R}^{2}}{P_{S}\left|h_{S R}\right|^{2}+\sigma_{D}^{2}}+\sigma_{D}^{2}
$$

The destination receives two variants of the original message:

$$
Y_{D}=W_{S D} Y_{S D}+W_{R D} Y_{R D}
$$

Assuming the knowledge of $h_{S R}$ and $h_{S D}$, the gain factors $W_{S D}$ and $W_{R D}$ are chosen to maximize the resulting SNR with:

$$
\begin{gathered}
W_{S D}=\frac{h_{S D}{ }^{*}}{\sigma_{D}^{2}} \\
W_{R D}=\frac{\sqrt{\frac{P_{r}}{P_{S}\left|h_{S R}\right|^{2}+\sigma_{D}^{2}}}}{\frac{P_{r}\left|h_{R D}\right|^{2} \sigma_{R}^{2}}{P_{S}\left|h_{S R}\right|^{2}+\sigma_{D}^{2}}+\sigma_{D}^{2}}(9) .
\end{gathered}
$$

Finally, the instantaneous mutual information of the system is expressed as:

$$
I_{A F}=\frac{1}{2} \log _{2}\left(1+\gamma_{S D}+\gamma_{R D}\right)(10)
$$

where $\gamma_{S D}$ and $\gamma_{R D}$ are the instantaneous SNR of the sourcedestination and relay-destination links, respectively, and are given by:

$$
\begin{gathered}
\gamma_{S D}^{A F}=\frac{P_{S}\left|h_{S D}\right|^{2}}{\sigma_{D}^{2}}(11) \\
\gamma_{R D}^{A F}=\frac{P_{S}\left|h_{R D} \beta h_{S R}\right|^{2}}{\left|h_{R D} \beta\right|^{2} \sigma_{R}^{2}+\sigma_{D}^{2}}=q\left(\frac{P_{S}\left|h_{S R}\right|^{2}}{\sigma_{R}^{2}}, \frac{P_{R}\left|h_{R D}\right|^{2}}{\sigma_{D}^{2}}\right)(12)
\end{gathered}
$$

with

$$
q(x, y)=\frac{x y}{x+y+1}
$$

We model both the communication channels between the relays and eNodeB and between the D2D devices and relays via small-scale Rayleigh fading with path loss and log-normal shadowing. We calculate the gain using $g=|h|^{2} d^{-\alpha}$, where $h$ is a Rayleigh random variable, $\alpha=4$ and $\mathrm{d}$ represents the distance between the transmitter and the receiver of the communicating pair.

The main objective of the proposed approach is to maximize the sum-rate of DUEs while guaranteeing the QoS of the relays. In game theory, each player competes to maximize its payoff presented by a utility function. Hence, each DUE tries to increase its throughput while consuming low energy. This tradeoff can be quantified as the ratio of the achieved throughput to the transmission power:

$$
u_{i}=\frac{T_{i}}{p_{i}}(14)
$$

where $T_{i}$ represents the throughput of the $i^{\text {th }}$ DUE and $p_{i}$ is the transmission power in the relays' cellular channel. The throughput is also related to $p_{i}$ via the $\operatorname{SNR} \gamma$.

The above problem aims to maximize the total energy efficiency of all links in the cell. This problem is centralized and NP-hard complex [15]; the eNodeB must know the full channel state information (CSI) of all links. Besides, it brings considerable overhead and information signaling. To address this issue, we investigate a distributed two stages Stackelberg approach.

The sellers represent the relays, and the buyers are the OCDUs. Each relay determines the price assigned to a bandwidth resource via the pricing function, which considers the current OCDU's numbers and demands. A relay prefers to sell throughput to the OCDU that provides the maximum price. The price depends on the DUEs number, the co-channel D2D locations, the required throughput, and the transmission powers.

The OCDUs compete to buy the maximum amount of resources. Each one aims to maximize its utility and minimize the cost at the same time. If the relay requests a high price, the OCDUs would ask for fewer throughputs or even decline their demands. The optimal price that maximizes both the relays and OCDUs utilities is reached at the Nash equilibrium.

We consider a system based on OFDMA. Each relay is allocated $\mathrm{w}(\mathrm{Hz})$ bandwidth for transmission. They share portions of their resource blocs with the OCDUs. Naturally, the data originating from the source (OCDUs) is divided into two parts: The first via the two-phase cooperative transmission and the second through the direct transmission from the source to the destination. However in out of coverage scenario, the source cannot reach the destination, so it sends its data only to the relay which is located in the coverage area of the source.

Let $w$ be the total bandwidth of the relay. If it decides to split $w_{i}\left(0 \leq w_{i} \leq w\right)$ bandwidth resources to the user i, the twophase relaying transmission occupies $w_{i}+w_{r}(\mathrm{~Hz})$ bandwidth of both the user $i$ and the relay. Consequently, a relay faces the problem of how much bandwidth to split with the OCDUs? How to divide its scarce resource between the competing users? And who favor?

\section{A. Proposed Stackelberg game model}

The relay device shares fractions of its bandwidth to backhaul the OCDUs' data. However, the relaying generates additional energy, calculation, and transmission delays. To tackle these issues, it sells this bandwidth to the device that pays the maximum price. Hence, it can utilize the cost to transmit its data in the future.

On the other hand, the OCDUs aim to obtain the highest profit i.e. maximizing their throughput and minimizing the price. Since the price depends on the strategies adopted by all the users, the resource competition is a strategic game. Consequently, both the relays and OCDUs should adjust their strategies until reaching equilibrium.

\section{B. Price update strategy of the relay node}

The price reflects the market quantity/price. i.e. if the bandwidth demand increases, the relay maximizes the price to get more benefits. If the demand decreases, it should attract more buyers to fit the deficiency. So, it reduces the charge. We propose a simple but tractable price strategy to charge the OCDUs as follows:

$$
c(w)=b\left(\sum_{i=1}^{N} w_{i}\right)
$$

where $b$ is a positive constant to ensurec $(w)>0, w=$ $\left\{w_{1}, w_{2}, \ldots, w_{n}\right\}$ denotes the set of strategies adopted by the OCDUs. 
The cost per unit of bandwidth resource is constrained by $c_{\text {min }}$ to ensure that the relay gets profit from the sale.

$\left(0 \leq w_{t h} \leq w\right)$ denotes the bandwidth proposed by the relay for cooperative transmission where:

$$
\sum_{i=1}^{N} w_{i} \leq w_{t h}
$$

If the total OCDUs demand exceeds the relay's resources, the user asking for the minimum bandwidth is omitted until the condition (16) is satisfied. The remaining OCDUs are charged by the same price, as they are similar to the relay.

\section{Utility function}

All the OCDUs in the game want to maximize their energy efficiency, by maximizing their throughput and minimizing the energy cost. If it transmits $L$ bits packed into a frame of $M F(M F>L)$ bits at a rate $R$ bits/s with a bandwidth $w$, its throughput can be expressed by:

$$
T=\frac{L}{M F} R f(\gamma)
$$

where

$$
f(\gamma)=[1-B E R(\gamma)]^{M F}
$$

is the probability of correct reception of a frame, we used the following approximate function:

$$
f(\gamma)=[1-2 B E R(\gamma)]^{M F}(19)
$$

In this paper, we employ

$$
B E R(\gamma)=\frac{4}{\log _{2} M} Q\left(\sqrt{\frac{3 \gamma \log _{2} M}{M-1}}\right)(20)
$$

We adopt 64-QAM as the modulation scheme.

$B E R(\gamma)$ is the bit error probability for coherent 64-QAM modulation. $R=\eta w$, where $\eta$ is the number of bit per symbol. In our case,$\eta=6$. Then (17) can be expressed as

$$
T=\frac{6 L}{M} w f(\gamma)
$$

The OCDU denoted $U_{i}$ utility depends not only on its preferences but also on the price proposed by the relay

$$
U_{i}=\frac{T_{s i, d i}\left(p_{i}, w_{i}\right)}{p_{i}+p_{r}}-c \cdot w_{i}
$$

where $p_{i}$ and $p_{r}$ are the transmission powers of the user $i$ and the relay respectively.

$$
T_{s i, d i}\left(p_{i}, w_{i}\right)=\frac{6 L}{M} w_{i} f\left(\gamma_{s i, d i}\right)
$$

The relaying price considers the total demand of the users. Consequently, the OCDU utility can be expressed by:

$$
U_{i}=\frac{6 L w_{i} f\left(\gamma_{s i, d i}\right)}{M\left(p_{i}+p_{r}\right)}-b\left(\sum_{j=1}^{N} w_{j}\right) w_{i}, \forall i \in M
$$

\section{Nash equilibrium of the game}

The Nash equilibrium is a strategy profile reached when no player can increase his utility by changing his strategy unilaterally, keeping all others' strategies unchanged. Let $w_{-i}=\left\{w_{1}, \ldots, w_{i-1}, w_{i+1}, \ldots, w_{N}\right\}$ be the set of strategies adopted by all OCDUs except $i$, and $w=w_{i} \cup\left\{w_{-i}\right\}$. Then user $i$ 's best-response function is given by:

$$
B_{i}(w)=\arg \max _{w_{i}} U_{i}\left(w_{i}, w_{-i}\right)
$$

The best strategies combination all the buyers, i.e. $w^{*}=\left\{w_{1}^{*}, w_{2}^{*}, \ldots, w_{N}^{*}\right\}$, determine the Nash equilibrium of the game if and only if:

$$
w_{i}^{*}=\max \left(0, B_{i}\left(w^{*}\right)\right), \forall i \in M
$$

To obtain the Nash equilibrium, we derive the utility function (24) with respect to $w_{i}$ and set each part to 0 as follows:

$$
\begin{aligned}
& \frac{\partial U_{i}}{\partial w_{i}}=\frac{6 L w_{i} f\left(\gamma_{s i, d i}\right)}{M\left(p_{i}+p_{r}\right)}-b\left(\sum_{j=1}^{N} w_{j}\right) w_{i} \\
& \frac{6 L f\left(\gamma_{s i, d i}\right)}{M\left(p_{i}+p_{r}\right)}-b\left(w_{i}+\sum_{j=1}^{N} w_{j}\right)=0
\end{aligned}
$$

The solution of these equations is the Nash equilibrium, i.e.

$$
w_{i}^{*}=\frac{3 L f\left(\gamma_{s i, d i}\right)}{b M\left(p_{i}+p_{r}\right)}-\frac{1}{2} \sum_{\substack{j=1 \\ j \neq i}}^{N} w_{j}^{*}, \forall i \in M
$$

\section{PROPOSED APPROACH: ENERGY EFFICIENT D2D COVERAGE EXTENSION ALGORITHM}

The proposed distributed Joint SE/EE Stackelberg algorithm is presented in algorithm 1. It operates with a minimum data broadcast. Each OCDU communicates only with the relay. It gets the information about the other OCDUs from the pricing rather than their exact strategies. Then, it adapts step by step its policy until reaching the Nash equilibrium. At the iteration $i t$, the strategy adopted by the $i^{t h}$ OCDU depends on its strategy taken at the previous iteration:

$$
w_{i}(i t+1)=B_{i}(w(i t))+w_{i}(i t) \frac{\partial U_{i}}{\partial w_{i}}, \forall i \in M
$$

If the amount of bandwidth allocated to the OCDU at the iteration it is less than the optimal, the OCDU changes to $\frac{\partial U_{i}}{\partial w_{i}}>$ $O$ at the next iteration, and the player increases its demand. Otherwise, if the allocated bandwidth to this player is higher than the optimal one, it changes to $\frac{\partial U_{i}}{\partial w_{i}}<0$ and decrease his demand at the iteration $i t+1$.

Finally, the Nash equilibrium is reached, when the following condition is satisfied for each OCDU:

$$
w_{i}(i t+1)=w_{i}(i t)=w_{i}^{*}, \forall i \in I
$$

To meet this condition, the optimal strategy of the game should insure $\frac{\partial U_{i}}{\partial w_{i}}=0$ for all the players in the game. 


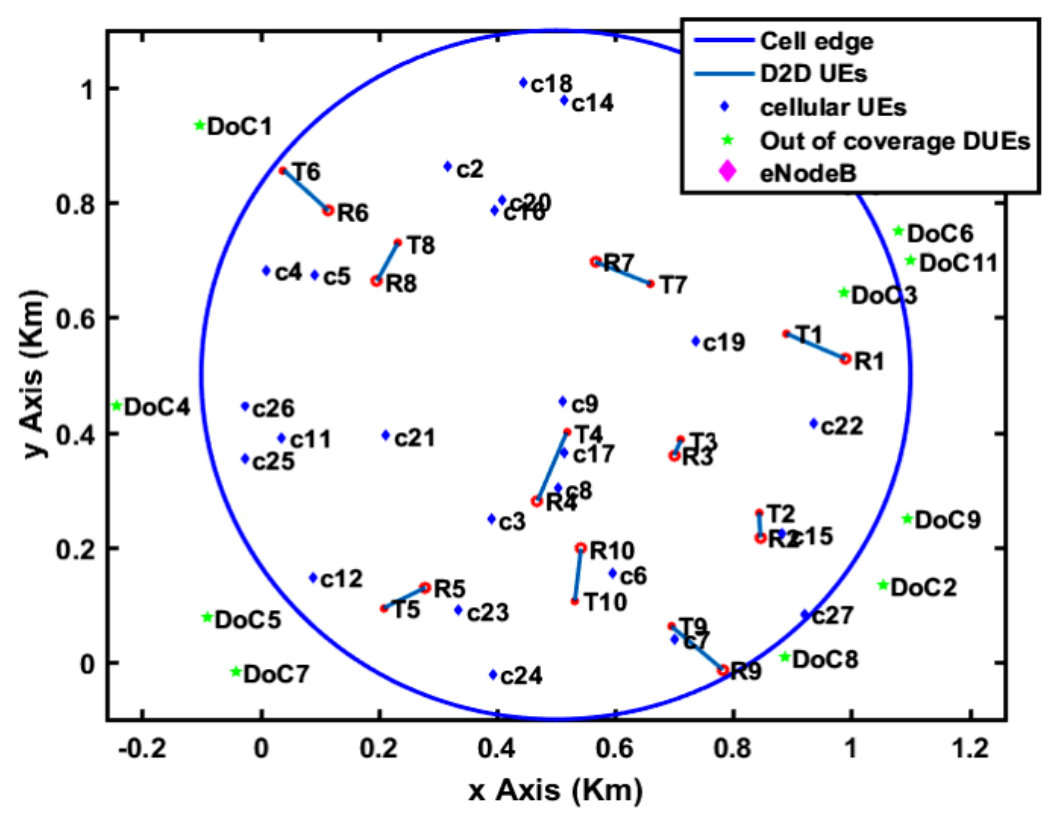

Fig. 2. The locations of $N$ CUEs and M D2D UEs: $(N=20, M=15$, the cell radius is $500 \mathrm{~m}$, max D2D distance $=50 \mathrm{~m})$

ALGORITHM 1: THE JOINT SE/EE STACKELBERG ALGORITHM

The Joint SE/EE Stackelberg algorithm

1: Assign the CUEs resource allocation vector $C R B=[1] x[\mathrm{k}]$

2: Variable $c^{*}=[0], n=1, i t_{i}=1, i t_{\max }=30, D=30, K=30$, $c_{r d}=500$ and $\Delta=10^{-3}$, dmax $=50$

3: Generate random distribution for $N$ cellular and $M D U E S$

4: While $n \leq M$ do;

5:Discover DUE relays: Drel[1][M-1]

6: Whileit $_{i} \leq i t_{\max } d o$;

7: $B_{i}(w)=\arg \max _{w_{i}} U_{i}\left(w_{i}, w_{-i}\right) \quad$ (equation 25$)$

8:if $w_{i}^{*}=\max \left(0, B_{i}\left(w^{*}\right)\right), \forall i \in I$ (equation 26)

9:then $N E[n][1]=\sigma^{i}$ And $n=n+1$;

10: else: $i t_{i}=i t_{i}+1$;

11: End while.

\section{Simulation RESUlts: ENERGY EFFICIENT D2D COVERAGE EXTENSION ALGORITHM}

To evaluate the performance of the proposed algorithm, we used Matlab as numerical computing environment. We have tested the software on intel core i7 machine on Microsoft Windows operating system. We provide a set of PPP distribution with intensities $\lambda_{D 1}, \lambda_{D 2}$ and $\lambda_{c}$ and vary the number of OCDUs and relays in the simulations.

We simulate our cellular environment formed by an eNodeB placed at the cell centre and a set of $\mathrm{N}$ cellular UEs distributed around. The initial transmission powers of the OCDUs are fixed to $23 \mathrm{dBm}$. We set the power of the eNodeB to ensure an acceptable SINR for any relay in the cell especially at the cell edge.
The following path loss model is used:

$$
P L(d)=P L\left(d_{0}\right)+10 \alpha \log (d)(d B)
$$

where $d$ represents the distance between the transmitter and the receiver, $P L(d)$ represents the receiver's path loss, $P L\left(d_{0}\right)$ is the reference path loss and $\alpha$ is the path loss exponent. It is considered the same for all the channels and assumed to 4 . Any cellular device inside the cell can be a potential relay for an out of coverage DUE if the distance and the channel conditions are satisfied. The relays and DUEs are distributed randomly and all D2D communications are constrained by $d_{\max }=50 \mathrm{~m}$.

In the figure 2, the locations of cellular and D2D UEs are generated randomly within and around a cell radius of $500 \mathrm{~m}$. direct D2D communications are presented by red dots, CUEs by blue diamonds and out of coverage DUEs (OCDUs) by green stars. The numbers above each UE represent every link's id and type (cellular, D2D, out of coverage DUE).

For the rest, Monte Carlo simulations are used to estimate the spectral and energy efficiency of D2D communications. Then simulation results are compared to other state of art solutions. To evaluate the performance of the proposed algorithm, and derive the optimal price and bandwidth to buy from the relay, we set the simulation parameters as follows: $\mathrm{L}=64, \mathrm{M}=70$ and $\mathrm{w}=5 \mathrm{MHz}$. In the first stage of the algorithm, each DUE fills its relay list according to its location; a relay can assist any OCDU located inside a disc of radius $d_{\text {max }}$ centered by itself.

Figure 3 shows the average price charged by the relays according to the total user's number. The presented results are averaged through 1000 Monte Carlo simulations. We observe that both the total and the average bandwidth demand of each user increase corresponding to the OCDUs number. 


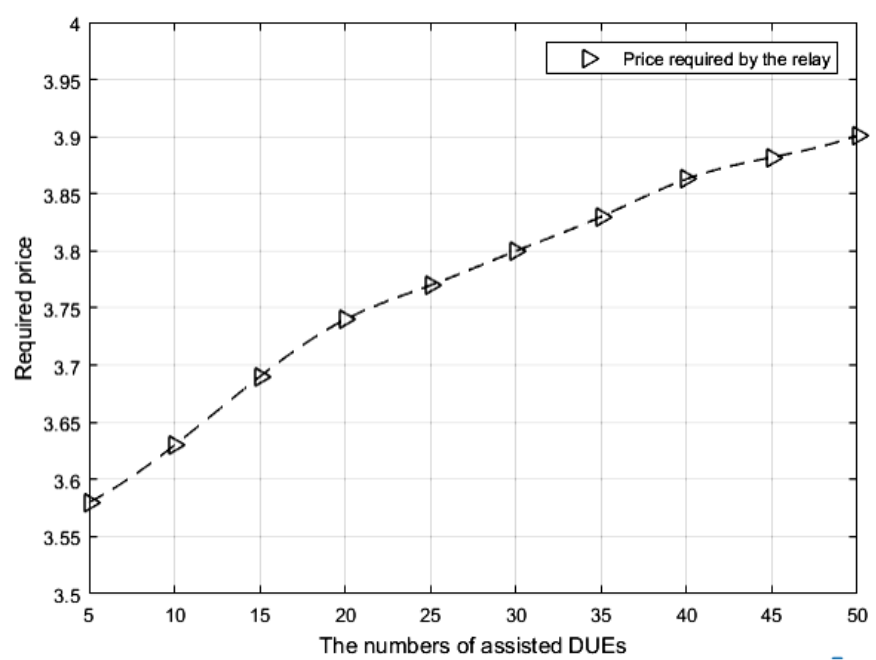

Fig. 3. The required price according to the distance between served DUEs and their relays

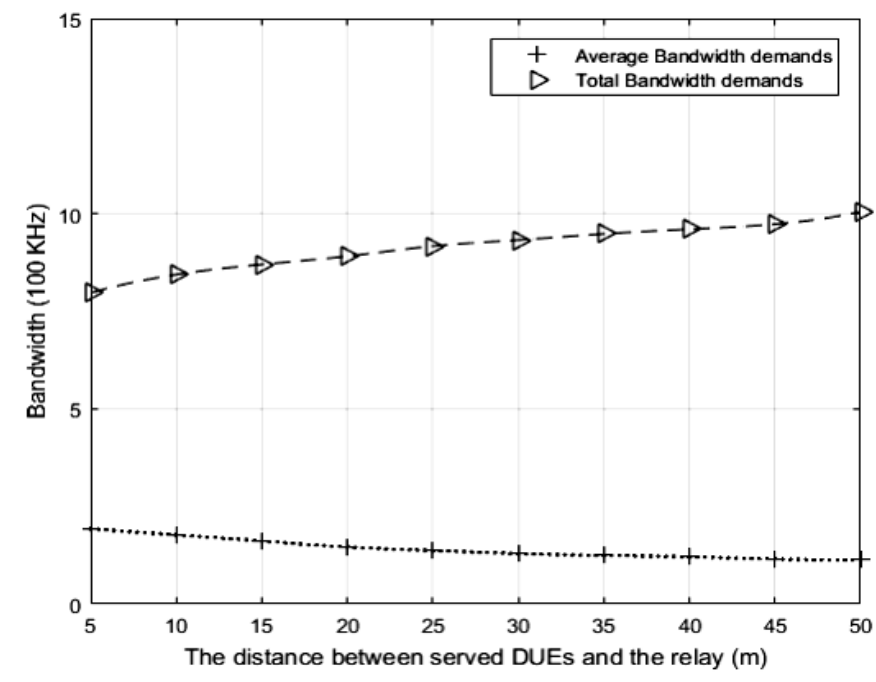

Fig. 4. Average and total bandwidth demands according to the distance between DUEs and their served relays

This assumption matches very well with the price function (15), where the price is proportional to the total bandwidth demand of the assisted users. This strategy is implemented to maximize the relays' profit; when the user demand decreases, the relay should reduce the charged price to get more buyers. Otherwise, the users would not purchase any bandwidth from the relay.

Figure 4 presents the amount of bandwidth bought by DUEs according to their distance to the relay. We observe that the average bandwidth allocated to a close OCDU is higher than that attributed to a far device. This reasoning is expected because a far away OCDU cannot absolutely get advantages from cooperative transmission. It has to spend more energy to transmit its data to the relay and may face inferior channel conditions. Otherwise, close devices maximize their profits without competing with the other buyers.

Both the price and the total users' demand reach their maximum when the OCDUs are close to the relay. Meanwhile, there are better channel conditions, so the bandwidth demands become more competitive. Accordingly, the price increases as

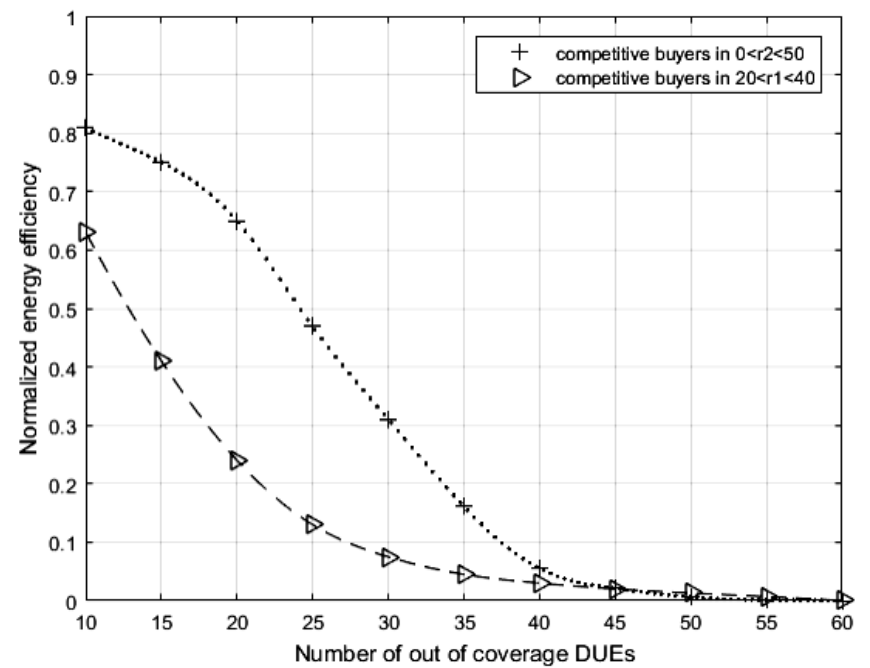

Fig. 5. The normalized energy efficiency corresponding to the number of competitive buyers in a specific area

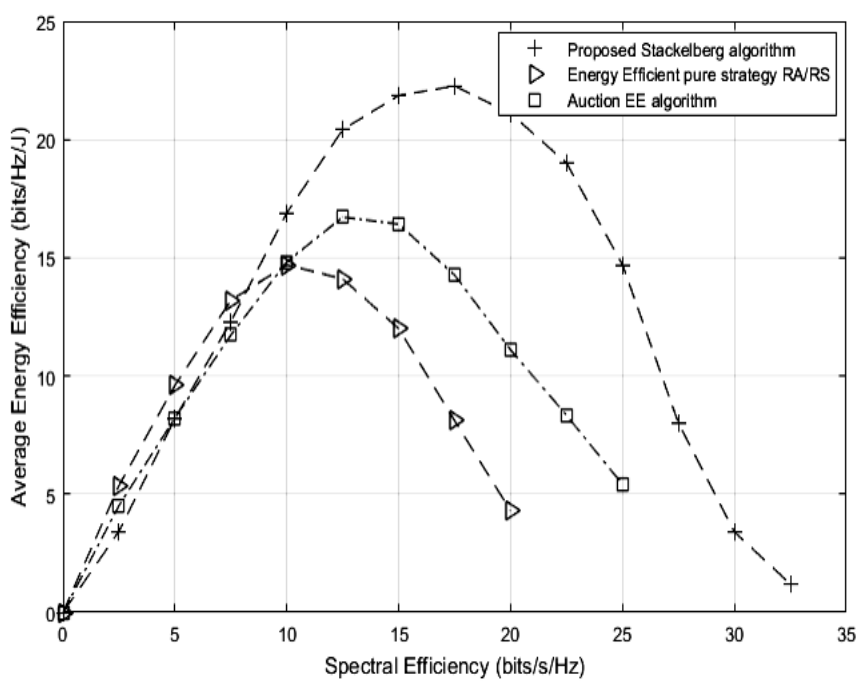

Fig. 6. The average energy efficiency corresponding to the spectral efficiency

expressed in equation (15), and the OCDUs improve their requests, even with a high price.

Figure 5 represents the average energy efficiency of all the out of coverage users. The results are averaged over a total number of 1000 Monte Carlo simulations. The energy efficiency is maximal when there are few competitive buyers. When the total OCDUs number decreases, the bandwidth demands decrease, which increases the utility function as expressed in equation (24). Two cases are plotted; in the first, all the competitive buyers are located in a cell-centered by the relay. Besides, they are all involved in the purchase. In the second, the buyers are located at a distance $20 \mathrm{~m} \leq \mathrm{d}_{\mathrm{r}} \leq$ $40 \mathrm{~m}$. Simulation results demonstrate that when there is a high device number around the relay, their channel conditions degrade due to the co-channel interference related to the distance between them. Consequently, they decrease their bandwidth demands even with a low price.

Meanwhile, the OCDUs located little far from the relay (at a distance $d_{r} \in[20,40]$ ) can continue increasing their demands for two reasons. First because the close users are not attracted to buy bandwidth resources, and second, because the price 
drop. (at a distance $d_{r} \in[20,40]$ can continue increasing its demand since the close users are not interested to buy bandwidth resources, as well as due the decreasing price.

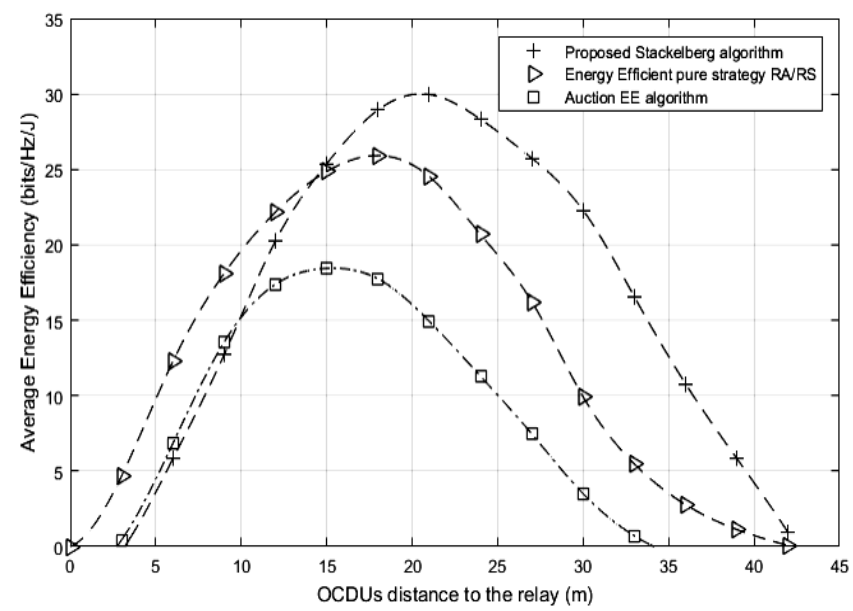

Fig. 7. The average energy efficiency corresponding to the OCDUs location

Figure 6 presents three different relay selection implementations; our proposed joint SE/EE Stackelberg algorithm, the Auction EE relay selection algorithm and the energy-efficient pure strategy algorithm. Comparing the obtained results, the proposed joint SE/EE outperforms the two mentioned algorithms in terms of maximum reached Energy efficiency $=23$ (bits/second/Joule) and spectral efficiency $\mathrm{t}=15$ (bits/second/Hz) versus 17 (bits/second/Joule) at $\mathrm{t}=12.3$ (bits/second $/ \mathrm{Hz}$ ) for the second and 15 (bits/second/Joule) at $\mathrm{t}=10$ (bits/second/Hz) for the last.

These results are expected because, in pure strategy games, each transmitter is self-interested. It competes to select the optimal relay without coalitions. Thus, multiple transmitters can simultaneously select the same relay, which causes harmful interferences and energy drop in its battery. The gain obtained by reducing the energy in the D2D transmitter is not always able to compensate for the additional power consumption at the relay. Moreover, in this algorithm, the OCDUs follow the same reasoning: allocating the quite near relay, which causes higher interferences and errors and leads to SE and EE loss. The auction algorithm provides mean results because the OCDUEs bid for the relay requiring the low energy transmission without considering the spectral efficiency requirements. This relay is typically the nearest one to the transmitter. Consequently, it influences the signal strength and further degrade the SE/EE tradeoff if the relay is selected simultaneously by many D2D.

Contrarily to these approaches, the proposed mixed Stackelberg game considers both the energy and the spectrum optimization. Each source and relay calculates the optimal amount of data and power to transfer, and then the relay chooses the communications that will serve. Besides, a D2D can select many relays simultaneously, which guarantees an acceptable price.

Whatever the relay selection criteria, the maximum achievable EE decreases monotonically if the SE increases more than a threshold $t$. These results are reasonable because maximizing the spectrum efficiency requires more energy from relays battery to route additional data to and from the eNodeB. It shows the importance of the power control step that privileges relays that don't require high transmission power.

Figure 7 shows the EE corresponding to the OCDUs locations. In the three plotted relaying strategies, we observe that the EE is maximal when the DUEs are located quite far from their serving relays, and there are few buyers in the relay transmission radius. These results match well with the pricing and the utility function. The closer the DUE to the relay, the low energy is required, but the higher co-channel interference occurs. Otherwise, the farthest OCDU requires more energy to route its information via cooperative communication. Besides, it may experience inferior channel conditions.

However, an OCDU located at the middle of the transmission radius gains more energy efficiency because both close and far devices are not interested in purchasing resources from the relay due to the former reasons: the significant interference and energy consumption requirements.

The second curve displays the energy-efficient pure strategy algorithm, and the third presents the auction EE algorithm. We observe inferior performances in both the maximum achievable energy efficiency and the distance to the relay. Whatever the relay selection strategy, the EE increases first and then decreases rapidly since each OCDU is allocated only one relay. Hence, it cannot split its bandwidth demands between a set of competing relays. So if it asks for high bandwidth while there is no relay satisfying the total request, it will be omitted. Besides, if it is far from the relay, it experiences poor channel conditions, spend more energy, and the relay will privilege the nearest devices. However, if it is very close, it will be affected by the interferences. That is why the EE typically achieves its maximum at the middle of the transmission radius.

There is always optimal power value and distance to preserve the spectral and energy efficiency gains. The energy mitigation decreases the interferences between the co-channel communications. Consequently, it increases the EE and SE gains. Yet, below the optimal value, it cannot compensate for the SE and EE loss caused by the useful signal gain drop. That is why the algorithm performs the best EE/SE values when the D2D transmitter and receiver are close to each other but far from the other buyers that may cause interference to their communication.

\section{CONCLUSION}

In this paper, we extended our previous work on inter-cell multi-hop D2D cluster by a new energy and spectral-efficient algorithm for coverage extension. We use a simple and tractable Stackelberg model to answer to public safety and emergency use cases. We evaluate the performance of the proposed approach in terms of energy efficiency, spectral efficiency, and the number of equipments in the cell. The simulation results demonstrate significant improvements compared to the existing state of art solutions. Based on the comprehensive analysis, we further identified eventual problems and challenges that deserve future research. We considered a traditional homogenous network in which all the users have the same utility function. However, the more challenging issues in a $5 \mathrm{G}$ network are the heterogeneous networks (Het-Nets) densification and the devices' mobility. 
So, more work is needed to integrate these criteria in the utility function. Moreover, the eNodeB transmits at a high power and generates significant interference to the close D2D communications. As a solution, we plan to proceed with a guard area around the eNodeB and suggest further extending the coverage through carrier aggregation and advanced RAN coordination for mid-band and high-band 5G deployments.

\section{REFERENCES}

[1] Sawsan, Selmi; Ridha, Bouallègue. Spectral and Energy Efficient D2D Communication Underlay 5G Networks: A Mixed Strategy Approach. Journal of Communications Software and Systems, [S.1.], v. 16 , n. 1, p. 57-65, apr. 2020. ISSN 1846-6079.

[2] Malik, Praveen \& Wadhwa, Deepinder \& Singh, Jaspal. (2020). A Survey of Device to Device and Cooperative Communication for the Future Cellular Networks. International Journal of Wireless Information Networks. 27. 10.1007/s10776-020-00482-8.

[3] J. Lyu, H. -M. Wang and K. -W. Huang, "Physical Layer Security in D2D Underlay Cellular Networks With Poisson Cluster Process," in IEEE Transactions on Communications, vol. 68, no. 11, pp. 7123-7139, Nov. 2020.

[4] Adnan MH, Ahmad Zukarnain Z. Device-To-Device Communication in 5G Environment: Issues, Solutions, and Challenges. Symmetry. 2020; 12(10): 1762

[5] Asadi, Arash \& Mancuso, Vincenzo \& Gupta, Rohit. (2016). An SDRbased Experimental Study of Outband D2D Communications. 10.1109/INFOCOM.2016.7524372.

[6] Hayat, O., Ngah, R. \& Mohd Hashim, S.Z. Full Duplex DD for In-Band D2D Communication. Wireless Pers Commun 115, 679-694 (2020).

[7] S. Selmi and R. Bouallègue, "Joint Spectral and Energy Efficient Multihop D2D Communication Underlay 5G Networks," 2020 International Conference on Software, Telecommunications and Computer Networks (SoftCOM), Split, Hvar, Croatia, 2020, pp. 1-6.

[8] E. M. Mohamed, B. M. Elhalawany, H. S. Khallaf, M. Zareei, A. Zeb and M. A. Abdelghany, "Relay Probing for Millimeter Wave Multi-Hop D2D Networks," in IEEE Access, vol. 8, pp. 30560-30574, 2020 Amodu, Oluwatosin \& Othman, Mohamed \& Noordin, Nor \& Ahmad, Idawaty. (2019). Relay-Assisted D2D Underlay Cellular Network Analysis Using Stochastic Geometry: Overview and Future Directions. IEEE Access. PP. 1-1. 10.1109/ACCESS.2019.2933915.

[9] Ghosh, Sayanti \& Mondal, Soumen \& Dhar Roy, Sanjay \& Kundu, Sumit. (2020). D2D Communication with Energy Harvesting Relays for Disaster Management. International Journal of Electronics. 107 10.1080/00207217.2020.1726488.

[10] Zhou, K., Gui, J. \& Xiong, N. Improving cellular downlink throughput by multi-hop relay-assisted outband D2D communications. J Wireless Com Network 2017, 209 (2017).

[11] Alsharfa, Raya M.; Mohammed, Saleem L.; Gharghan, Sadik K.; Khan, Imran; Choi, Bong J. 2020. "Cellular-D2D Resource Allocation Algorithm Based on User Fairness" Electronics 9, no. 3: 386.

[12] Yaacoub E. Cooperative energy efficient D2D clustering in LTE-A with enhanced QoS. Telecommun. Syst. 2018;9:1-14.

[13] Huo, Y., Xu, M., Fan, X. et al. A novel secure relay selection strategy for energy-harvesting-enabled Internet of things. J Wireless Com Network 2018, 264 (2018)

[14] Shen Zhang, Yi Peng,D2D Communication Relay Selection Algorithm Based on Game Theory, Procedia Computer Science, Volume 166,2020,Pages 563-569,ISSN 1877-0509.

[15] Zhang, Chuang \& Zhao, Honglin \& Jia, Min. (2015). A pricing-based cooperation bandwidth allocations algorithm using non-cooperative game theory. International Conference on Signal Processing Proceedings, ICSP. 2015. 2379-2384. 10.1109/ICOSP.2014.7015420.

[16] Chen, Long, W. Jigang and X. Zhang. "TARCO: Two-Stage Auction for D2D Relay Aided Computation Resource Allocation in HetNet." IEEE Transactions on Services Computing 14 (2021): 286299.

[17] Zhang, Shen \& Peng, Yi. (2020). D2D Communication Relay Selection Algorithm Based on Game Theory. Procedia Computer Science. 166. 563-569. 10.1016/j.procs.2020.02.020.

[18] Wang, Jingjing \& Jiang, Chunxiao \& Bie, Zhi \& Quek, Tony Q.S. \& Ren, Yong. (2016). Mobile Data Transactions in Device-To-Device
Communication Networks: Pricing and Auction. IEEE Wireless Communications Letters. 5. 300-303. 10.1109/LWC.2016.2547395.

[19] P. Li, S. Guo and I. Stojmenovic, "A Truthful Double Auction for Device-to-Device Communications in Cellular Networks," in IEEE Journal on Selected Areas in Communications, vol. 34, no. 1, pp. 71-81, Jan. 2016.

[20] Zhang, Y. and F. Li. "Efficiency Intra-Cluster Device-to-Device Relay Selection for Multicast Services Based on Combinatorial Auction.” Algorithms 8 (2015): 1129-1142.

[21] Jehan Zeb, Aamir Hassan, Muhammad Danish Nisar, Joint power and spectrum allocation for D2D communication overlaying cellular networks, Computer Networks, Volume 184, 2021, 107683,

[22] xu, Lei \& Junhao, Feng \& Biyao, Huang \& Zhou, Zhenyu \& Mumtaz, Shahid \& Rodriguez, Jonathan. (2017). Joint Relay Selection and Resource Allocation for Energy-Efficient D2D Cooperative Communications Using Matching Theory. Applied Sciences. 7. 491. 10.3390/app7050491.

[23] Cong, L., Zhao, L., Yang, K., Zhang, H. and Zhang, G. (2011), A Stackelberg game for resource allocation in multiuser cooperative transmission networks. Wirel. Commun. Mob. Comput., 11: 129141. https://doi.org/10.1002/wcm.922

[24] Y. Mao, J. Zhang, S. H. Song and K. B. Letaief, "Stochastic Joint Radio and Computational Resource Management for Multi-User Mobile-Edge Computing Systems," in IEEE Transactions on Wireless Communications, vol. 16, no. 9, pp. 5994-6009, Sept. 2017.

[25] Feng, G., Qin, X., Jia, Z. et al. Energy efficiency resource allocation for D2D communication network based on relay selection. Wireless Netw (2020). https://doi.org/10.1007/s11276-019-02240-y

[26] Wang, R., Cheng, D., Zhang, G., Lu, Y., Yang, J., Zhao, L., et al.(2017). Joint relay selection and resource allocation in cooperative device--to-device communications. AEU-International Journal of Electronics and Communications, 73, 50-58.

[27] Wang, R., Liu, J., Zhang, G., Huang, S., \& Yuan, M. (2017). Energy efficient power allocation for relay-aided D2D communications in 5G networks. Chin. Commun., 14, 54-64. 30.

[28] Rahman, M., Lee, Y. D., \& Koo, I. (2018). Energy-efficient power allocation and relay selection schemes for relay-assisted $\mathrm{d} 2 \mathrm{~d}$ communications in $5 \mathrm{~g}$ wireless networks. Sensors, 18(9), 2865.

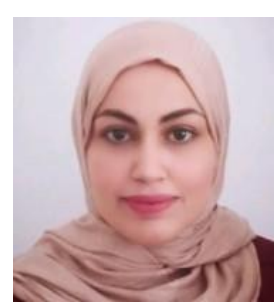

Sawsan Selmi is a computer science engineer. She received her master's degree in telecommunication from the National school of engineers of Tunis (ENIT) Tunisia, and she is currently a Ph.D. student at the Innov'Com Laboratory, the higher school of communication of Tunis (Sup'Com) Ariana, Tunisia. Her research interests include new generations of mobile networks, D2D communications, interference mitigation, energy, and spectral efficiency.

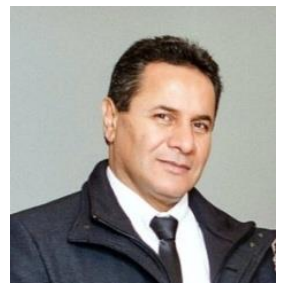

Ridha Bouallègue is Professor at ENIT, Tunisia and the director of Innov'COM Laboratory at Sup'Com Ariana, Tunisia. He received his Engineering degree, $\mathrm{Ph} . \mathrm{D}$, and the accreditation to supervise research (HDR) in telecommunication systems from the National School of Engineers of Tunis (ENIT), Tunisia in 1990, 1994, and 2003 respectively. His current research interests include mobile and satellite communications, access techniques, intelligent signal processing, CDMA, MIMO, OFDM, and UWB. 